\title{
Diacronie
}

Studi di Storia Contemporanea

$N^{\circ} 16,4 \mid 2013$

Le monarchie nell'età dei nazionalismi

\section{La monarchie imaginée : sur le royalisme dans l'idéologie de l'Action Française}

\section{Andrea Cavaletto}

\section{(2) OpenEdition}

\section{Journals}

\section{Édition électronique}

URL : http://journals.openedition.org/diacronie/875

DOI : 10.4000/diacronie.875

ISSN : 2038-0925

Éditeur

Association culturelle Diacronie

\section{Référence électronique}

Andrea Cavaletto, «La monarchie imaginée : sur le royalisme dans l'idéologie de l'Action Française », Diacronie [En ligne], № 16, 4 | 2013, document 5, mis en ligne le 01 décembre 2013, consulté le 20 avril 2019. URL : http://journals.openedition.org/diacronie/875; DOI : 10.4000/diacronie.875 


\title{
Diacronie
}

\section{$5 /$}

\section{La monarchie imaginée: sur le royalisme dans l'idéologie de l'Action Française}

\author{
Andrea CAVALETTO*
}

Cet article porte sur les liens entre nationalisme et royalisme au sein de l'Action Française. De quelle façon ce mouvement, qui ne comptait qu'un seul royaliste dans ses rangs lors de sa naissance, parvint à devenir peu de temps après le mouvement royaliste par excellence? En analysant plusieurs aspects de la construction idéologique a posteriori de la monarchie élaborée par Maurras, désavouée à la fois par les anciens royalistes et par les prétendants au trône eux-mêmes, nous tentons ici d'expliquer pourquoi cette image du Roi était bien loin d'être une simple nostalgie anachronique du passé monarchique. En accord avec les thèses de Brian Jenkins et Zeev Sternhell d'après lesquels l'Action Française était «populiste-moderne» plutôt que «élitisteréactionnaire» - l'article explique dans quelle mesure et par quelles voies la monarchie est devenue pour le mouvement une option de plus en plus symbolique pour la création d'un imaginaire de la nation alternatif à celui créé par la IIlème République: un outil conceptuel "nécessairement dynamique et créatif», subordonné aux exigences du nationalisme intégral.

\section{Introduction}

Les idées ne sont jamais tout à fait séparables des choses ${ }^{1}$.

Marc Bloch

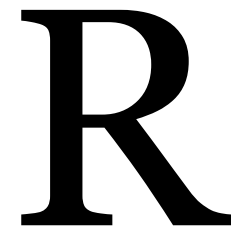
ené Rémond n'avait aucun doute lorsqu'il plaçait l'Action Française dans l'héritage des droites françaises du XIX siècle. D'après le célèbre historien et politologue, il s'agissait d'un mouvement qui était issu des trois

\footnotetext{
1 BLOCH, Marc, Les rois thaumaturges, cité dans ZUNINO, Pier Giorgio, L'ideologia del
} fascismo. Miti, credenze e valori nella stabilizzazione del regime, Bologna, Il Mulino, 1985, p. I. 
traditions consolidées dans l'histoire de la France moderne et qui, finalement, en étant rien d'autre qu'une «synthèse », n'apportait rien de nouveau sur scène. Élitisteréactionnaire et conservatrice selon les schémas traditionnels, l'AF demeurait dans l'analyse de Rémond tout à fait étrangère à l'ambiance contemporaine, étrangère aux problèmes de la modernité, reléguée dans son monde des idées où c'était possible de cultiver ses nostalgies royalistes ${ }^{2}$. Une telle relecture globale de l'histoire des droites françaises, qui n'a pas entraîné seulement des conséquences dans le cas de l'Action Française mais qui a visé (en le banalisant) tout mouvement d'extrême-droite ayant opéré en France pendant les premiers quatre décennies du siècle passé (y compris les ligues de l'entre-deux-guerres), est encore dominante aujourd'hui en France : ça à travers l'œuvre de ceux qu'on a souvent appelé «les gardiens du temple de la Rémondie»3. La recherche d'un supposé fascisme authentique (italien et allemand) emmène encore à présent chacun des héritiers de l'école rémondienne à qualifier $a$ priori tout autre phénomène d'extrême-droite dans l'Europe de la première moitié du XXème siècle comme essentiellement conservateur, traditionnel, antimoderne, ainsi que marginale, les véritables laboratoires politiques à la racine de la «réaction révolutionnaire» 4 ayant trouvé citoyenneté ailleurs en Europe. Par le biais d'un tel approche, caractérisé par un déterminisme téléologique et une attention insuffisante au contexte historique, Vichy ne pouvait qu'apparaître en tant que exception, événement ayant subi une «causalité exogène» (la défaite militaire) ${ }^{5}$. Le soi-disant «traditionalisme conservateur» de l'Action Française, marque de spécificité de l'extreme-droite en Héxagone, était lié d'une façon téléologique à la «brusque et anachronique remontée du passé» sous Vichy, où selon Rémond se déroulait «l'antimodernisme érigé en mode de gouvernement et en système social» 6 . Dans ce cadre, l'Action Française, avec son prétendu royalisme gardé à travers les décennies, se limitait à jouer le rôle de feu d'artifice trop prolongé. Maurras et ses camarades y

2 REMOND, René, La droite en France de 1815 à nos jours, Paris, Ed. Montaigne, 1963, pp. 180187.

3 Voir notamment à ce propos: RÉMOND, René, STERNHELL, Zeev, «Y a-t-il un fascisme français?», in Terre Humaine, 7-8/1952, pp. 37-47; SIRINELLI, Jean-François, Histoire des droites en France, t. I, Politique, Paris, Gallimard, 1992; MILZA, Pierre, La notion de 'préfascisme' est-elle pertinente?, in Bulletin du centre d'histoire de l'Europe du vingtième siècle, 4, 1990, pp. 7-50; ORY, Pascal, Du fascisme, Paris, Perrin, 2003; WINOCK, Michel, Nationalisme, antisémitisme et fascisme en France, Paris, Seuil, 2004; ID., Histoire de l'extrême-droite en France, Paris, Seuil, 1993.

4 WINOCK, Michel, Nationalisme, antisémitisme et fascisme en France, Paris, Seuil, 2004, pp. 193-195.

5 DOBRY, Michel, La thèse immunitaire face aux fascismes. Pour une critique de la logique classificatoire, in ID. (sous la direction de), Le mythe de l'allergie française au fascisme, Paris, Alban Michel, 2003, p. 33.

6 RÉMOND, René, Les droites en France, Paris, Aubier, 1954, p. 237. 
figuraient, au final, de «débris sociaux de l'époque préindustrielle toujours moins nombreux»7, et le mouvement dans son ensemble y était dépeint comme rien d'autre qu'un combat d'arrière-garde désespéré. Le fait que l'AF ait survécu longtemps s'expliquerait en définitive, d'après Rémond et ses élèves, par les «structures géologiques profondes» du tempérament politique ${ }^{8}$ et par l'immobilisme de la France et de sa «société bloquée»9. Pourtant, l'histoire de l'Action Française peut-elle vraiment justifier une telle lecture de l'idéologie du mouvement? Ces royalistes ne songeaient-ils vraiment qu'à un passé perdu à jamais? Nous proposons une contribution au débat sur le lien entre monarchies et nationalismes en reparcourant rapidement l'histoire de l'enracinement social et du développement doctrinal du mouvement royaliste «par excellence» pendant ses premières années de vie: cela en s'appuyant principalement sur les ouvrages de ceux qui ont sorti du folklore l'emprise politique de Charles Maurras et de ses camarades pour nous la restituer dans toute sa complexité.

\section{Avant l'Action Française: le déclin du courant monarchique}

Marc Bloch, dans un célèbre passage, nous expliquait que lorsque Charles X remit en vigueur l'ancienne cérémonie du couronnement à Reims en 1825, tout comme avant (y compris la cérémonie des guérisons miraculeuses) «à peine cent vingt personnes vinrent se faire guérir des écrouelles par la main royale». Lors du dernier couronnement, en 1774, «il y en avait $2.400 »^{10}$. La Révolution semblait avoir détruit une fois pour toutes l'édifice précédent: malgré les vœux des grands penseurs contrerévolutionnaires, gardiens de l'ordre imposé sur les champs de bataille et reconstitué à Vienne, c'était clairement impossible de revenir simplement en arrière, comme si rien ne s'était passé. D’ailleurs, une reconstruction a posteriori d'un passé prérévolutionnaire idyllique était vouée à l'échec si on pense que jadis, déjà, il y avait parmi les ennemis de 1789 ceux qui, comme De Maistre, dans leur critique des coutumes n'épargnaient même pas l'Ancien Régime: considéré coupable d'avoir engendré la punition révolutionnaire par sa décadence morale et politique, il avait été déjà épouillé du mythe d'une monarchie à restaurer telle qu'elle était avant.

\footnotetext{
7 JENKINS, Brian, L'Action Française à l'ère du fascisme: une perspective contextuelle, in DOBRY, Michel (sous la direction de), op. cit., p. 107.

8 REMOND, René, op.cit., pp. 186-187.

9 HOFFMANN, Stanley, Paradoxes de la communauté politique française, in ID. (sous la direction de), A la recherche de la France, Paris, Seuil, 1963, p. 26.

${ }_{10} \mathrm{BLOCH}$, Marc, Les Rois Thaumaturges. Étude sur le caractère surnaturel attribué à la puissance royale particulièrement en France et en Angleterre, Paris, Armand Colin, 1924, pp. 402-404.
} 
Ensuite, l'esprit de synthèse promu par la Charte sous la restauration, l'échec de la tentative ultra de Charles $\mathrm{X}$ et même de la monarchie libérale d'un Guizot, bref, l'ensemble des expériences politiques de la France des années 1800 vit bientôt la possibilité d'un retour à l'Ancien Régime s'éloigner toujours davantage. Les louvoiements des Prétendants et la mort du courant monarchique constitutionnel au début de la Troisième République avaient renvoyé les royalistes dans une marginalité politique et dans une doctrine légitimiste et autoritaire ${ }^{11}$.

Après l'aventure bonapartiste et Sedan, et suite à l'échec de la tentative du général Mac Mahon, un nouveau souverain faisait son entrée en scène, en guise de monarque abstract et tout-puissant. La III République, en équivalent laïque de l'Église, démontra pendant ses premières quinze années de vie d'être capable de savoir se consolider, produire une tradition nationale cohérente, en obtenant l'appui d'une petite et moyenne bourgeoisie toujours plus nombreuse et en reléguant ses ennemis aux marges, persécutés jusqu'à les rendre inoffensifs. La création d'une puissante religion civile par le biais des commémorations publiques et des nouveaux monuments publiques, une instruction publique marquée idéologiquement, bref, toute pratique collective guidée par en haut et finalisée à l'unité de la communauté nationale, à la « communion » des individus libres dans la nouvelle société bourgeoise, avait fusionné ensemble les concepts de Nation et République en plaçant cette dernière à l'abri des événements conjoncturels.

Les défaits, les partisans du Roi, continuaient de leur côté sur la voie d'une bataille vouée à l'échec: parmi les royalistes «classiques» restés fidèles au monarque, les idées de Coup d'État, «longuement discutées, parfois préparées mais toujours reportées au lendemain» tenaient lieu de seule possibilité pour une restauration rapide ${ }^{12}$, à défaut d'une véritable stratégie politique. Après 1892 le royalisme semblait être désormais devenu «une cause perdue» en France: les grandes familles d'affaires et de finance avaient transféré presque définitivement leur intérêt et leur allégeance à la République. C'est significatif, à ce propos, l'image du milieu royaliste à cette époque que nous donne Montesquiou, ce qui sera l'un des premiers membres du Comité d'Action Française : on $\mathrm{y}$ voit un Paris «jeune et bienpensant» où des hommes de bonne famille ayant reçu en héritage l'amour pour la couronne et un certain esprit de revanche «se plaisaient à conspirer entre minuit et quatre heures du matin, chez Maxim's ou dans quelques

${ }^{11}$ KUNTER, Tony, Charles Maurras: la Contre-Révolution pour héritage, Paris, Nouvelles éditions latines, 2009, pp. 30-40.

12 Ibidem, p. 31. 
restaurant de nuit»13. Rien de mieux, on dirait, que la «République de fils à papa», le "patriciat avoué» 14 sur lequel les jeunes royalistes s'acharnaient quotidiennement avec leur violence esthétique, en tant que derniers gardiens d'un monde pur et disparu. Si d'un côté les royalistes de la vieille école pensaient que la restauration monarchique pouvait se faire à la façon de celle de Charles II d'Angleterre «par quelque général Monk, qu'il convenait d'éduquer en ce sens»15, de l'autre côté ils manquaient d'une quelconque stratégie et dans l'attente du bon moment ils peinaient à promouvoir une doctrine attirante et novatrice. D'abord le bonapartisme et ensuite le boulangisme, d'ailleurs, avaient déjà démontré à suffisance que la réaction, c'est-à-dire l'évangile de l'ordre et la haine de la démocratie bourgeoise, n'avait guère besoin d'un Roi au temps du nationalisme et du culte de l'Armée, les chefs invoqués pouvant arriver d'ailleurs donc pas forcement de la maison de Bourbon-Orléans.

Une fois tombée les schémas traditionnels, un nouveau laboratoire politique était tout prêt à déclencher son potentiel créateur: l'Affaire Dreyfus fit bientôt le reste, en divisant la nouvelle-née «opinion publique » en deux camps opposés, «la Raison d'État d'un côté, l’idée des droits de l'homme et de la liberté individuelle de l'autre» ${ }^{16}$. Ce tournant historique, qui a eu tant de conséquences sur les événements du XXème siècle ainsi que sur le présent, était bien loin - évidemment - d'être un duel entre la République et le Duc d'Orléans, le «brouillement des frontières» à droite ayant été accompli une fois pour toutes. Si c'est vrai, en effet, que lors de l'Affaire les nationalistes mirent en cause pour la première fois la légitimité du régime au pouvoir, la question - politique et culturelle - posée par les antidreyfusards dans leur intégralité ne portait plus désormais sur la forme du régime, mais d'abord sur la définition du régime existant: quelle République faut-il à la France ? Quant aux monarchistes dans leur ensemble, ils semblaient avoir raté le dernier train: ils n'avaient pas su barrer la route aux dreyfusards et même pas se faire admettre en tant que membres du «Parti du Roi» dans l'hétéroclite coalition nationaliste. La place était dès lors toute prête pour l’Action Française.

\footnotetext{
${ }^{13}$ Lettre de Montesquiou à Maurras, 22 août 1900, parue dans CALLU, Gillet, Lettres à Charles Maurras, Paris, Septentrion Presses Universitaires, 2008, pp. 41-42.

14 MAURRAS, Charles, L'avenir de l'intelligence, dans ID., Euvres capitales, Paris, Flammarion, 1959, pp. 110-111.

${ }_{15}$ NATTER, François, ROUSSEAU, Claude, Introduction à MAURRAS, Charles, De la politique naturelle au nationalisme intégral, Paris, Natter, 1972, p. 11.

${ }^{16}$ COLETTE CAPITAIN, Peter, L’idéologie de l’Action Française, Paris, Seuil, 1972, p. 7.
} 


\section{La naissance}

Eric Hobsbawm nous a bien appris que toute tentative de restaurer un passé perdu «ne peut littéralement pas aboutir, sauf sous des formes triviales» ${ }^{17}$. Ça pourrait étonner, pourtant la réflexion de l'historien anglais aurait pu être souscrite sans aucun doute par les fondateurs du Comité d'Action Française, né dans le marasme réactionnaire de l'Affaire Dreyfus et de l'expérience décevante de la Ligue de la Patrie Française. Après l'échec aux élections de 1898, Henri Vaugeois et Maurice Pujo dans une lettre à «L'Eclair» soulignaient vouloir «refaire de la France républicaine et libre un État organisé à l'intérieur, puissant à l'extérieur, comme sous l'Ancien Régime », pourtant «sans recourir aux formes du passé» car, affirmaient-ils, «on ne fait rien avec les morts» ${ }^{18}$. Ils avaient beau, du reste, éprouver de la sympathie pour la famille d'Orléans, étant donné que dans le milieu d'où ils étaient issus on estimait, en majorité, que la République serait durable et la Restauration impossible.

Ils étaient, en effet, des jeunes hommes sans aucun doute républicains d'éducation et d'aspiration, ceux qui se réunissaient en 1899 au Café de Flore autour du Comité: parmi eux ne se distinguait qu'un seul monarchiste avoué, Charles Maurras. Si Pujo peu de temps auparavant flirtait avec l'anarchie et Daudet avait grandi dans la bonne société des Zola, Berthelot et Hugo, Vaugeois de son côté avait un passé socialiste et Montesquiou était un catholique qui s'était récemment rallié à la Troisième République. François de Mary, ce qui serait devenu le premier président de l'AF, se présentait de républicain convaincu. Bref, à ses débuts, le groupe passait pour un mouvement nationaliste parmi les autres. D'abord, il faut souligner que cette organisation politique n'aurait pu exister si l'Affaire n'avait pas mêlé toutes les positions politiques traditionnelles: le besoin d'ordre, d'autorité, de force gouvernementale se fondait dans le cour de ces jeunes avec le besoin de liberté, de progrès, d'ouverture vers l'avenir. Si le boulangisme avait été, de ce point de vue, une antichambre du nouveau laboratoire politique de la droite française, l'Affaire ouvrait davantage les portes à expériences politiques tout à fait originales.

Le choix royaliste paraît encore bien loin d'être pris pour les futurs compagnons de route de Maurras: lors du premier rencontre entre l'écrivain provençal et Vaugeois, ce dernier défendait encore opiniâtrement les droits de l'individu et l'héritage révolutionnaire, convaincu qu'on pouvait concilier le pur patriotisme avec les idées

17 HOBSBAWM, Eric, L’invention de la tradition, Paris, Ed. Amsterdam, 2006, pp. 16-17 [éd. originale: The invention of tradition, Cambridge, Cambridge University Press, 1983].

18 WEBER, Eugen, op. cit., p. 35. 
républicaines. Même l'accuse portée contre la Troisième République par la majorité de ces jeunes était indice de la présence d'intraitables valeurs républicaines. Les hommes du Comité qui venait de naître ne lui reprochaient, en effet, que d'être «faible et anarchique sans être libérale»19; une accusation qui n'avait encore aucun lien de parenté avec la mise en cause radicale des valeurs de 1789. En outre, le niveau d'étrangeté de ces jeunes hommes par rapport aux vieux milieux fidèles au Roi apparaît dans toute sa clarté si on constate que jusqu'au 1905 nul rapport de police consacré à l'activité des réseaux monarchistes aurait contenu un seul mot sur l'Action Française. Comment peut-on expliquer donc la transformation de ce qui avait été d'abord un mouvement républicain patriote et antidreyfusard en un mouvement royaliste, le plus sui generis et le plus victorieux des mouvements royalistes du XXème siècle, devenu pour la France entière «le grand centre didactique de la droite, [...] tenant lieu de référence et faisant autorité également pour une masse conservatrice à laquelle il manquait une doctrine»20? Autrement dit, où se situe la transition idéologique, sociale et politique d'un mouvement qui était formé de républicains antidreyfusards vers une organisation prête à remplacer les vieux dirigeants royalistes, et quel lien avait-il été gardé avec ce dernier, du point de vue idéologique, culturel et stratégique?

Pour obtenir enfin une réponse, peut-être toujours partielle mais satisfaisante à ces questions, l'historien d'aujourd'hui devrait au moins se méfier des parcours trop linéaires, ou bien trop drastiques, et surtout des idées reçues. Le paradigme traditionnel, «rémondien», jugée trop apaisante à l'égard de l'histoire des droites en amont et sous le régime, a été mis en cause par plusieurs historiens étrangers seulement à partir des années 1970, lors de l'avènement de la «Révolution paxtonienne»: bouleversant d'un façon inouïe le milieu académique français et déchirant toute idée reçue sur Vichy et la collaboration, mais aussi sur le paysage et sur l'évolution de l'univers des droites françaises pendant la première moitié du XX siècle, l'œuvre de l'historien américain a ouvert la voie à celles qu'avant l'on jugeait d'hérésies académiques. Dès lors, Robert O. Paxton, Ernst Nolte, Zeev Sternhell et Brian Jenkins ont essayé, chacun à sa façon, de ébranler les fondements du récit «paroissial» de ce qu'on appelle la «thèse immunitaire» pour analyser en profondeur les parcours des droites françaises au-delà des vieilles frontières, décryptant les idées politiques qui en ont jailli à partir de $1890^{21}$. Pour ce qui concerne l'Action Française, ils lui ont restitué

\footnotetext{
19 Ibidem, pp. 37-44.

20 ROGGER, Hans, WEBER, Eugène (sous la direction de), The European Right: a Historical Profile, London, Weidenfield and Nicolson, 1965, p. 97.

21 Voir notamment sur la «Révolution paxtonienne»: FISHMANN, Sara et al., La France sous Vichy : autour de Robert O. Paxton, Paris, Ed. Complexe, 2004.
} 
un statut d'appartenance à plein titre à son époque, en y voyant un mouvement tout à fait moderne, hégémonique à droite sur le plan culturel et ancêtre, sinon précurseur, des ligues fascistes ou fascisantes de l'entre-deux-guerres, auxquelles elle a transmis une partie de son héritage. Pour résumer, la faute aux défenseurs de la thèse immunitaire a été pour eux, entre autres, de minimiser la présence d'éléments rétrogrades dans les idéologies fasciste et nazi, en séparant d'une façon très nette les deux plus grands exemples historiques de «réaction révolutionnaire» des autres phénomènes plus ou moins contemporains. Le royalisme de l'Action Française, jusquelà considéré comme la preuve d'un incontestable ancrage dans le passé du mouvement et cause primaire de son échec politique, a été analysé d'une façon tout à fait nouvelle. Si on a le droit de dire qu'un fait de mentalité est d'abord, en lui-même, un fait d'histoire, le nationalisme intégral néo-royaliste de l'Action Française, jamais réalisé pourtant souvent victorieux au niveau politique, intellectuel et culturel, a occupé un espace très important tout au long d'une époque de crise et de mutation fondamentale pour l'histoire française et européenne, en continuant à exercer son rôle et son influence un peu partout dans les milieux de la droite. Plus de trente ans après sa naissance, suite aux émeutes antiparlementaires du 6 février 1934, écrit Weber, l'Action Française «se trouva presque l'incarnation de ce qui pouvait être l'esprit du temps [...] et c'était dans l'Action Française que l'opposition au Front Populaire devait trouver ses idées» ${ }^{22}$. Il s'agit sans doute d'un but qu'une organisation totalement déconnectée de la réalité du monde moderne et lié au mot d'ordre de la restauration nostalgique de la vieille monarchie d'Ancien Régime, telle que la configurait Rémond, aurait eu mal à achever. Ça n'aurait pas été possible, d'ailleurs, si dans la compétition sauvage née à l'extrême droite de l'échiquier politique ce petit groupe nationaliste né de l'Affaire n'avait façonné sa propre marque originale, destinée à une longue vie. Cette marque, douée d'une puissance symbolique, était le néo-royalisme : un royalisme totalement transformé et mis au service d'un système de pensée nouveau et moderne, prêt à s'acharner sur les ennemis du XX siècle. C'est là qui fait sa sortie en scène l'homme qui devient bientôt le chef incontesté de l'Action Française, Charles Maurras.

22 WEBER, Eugène, op. cit., p. 388. 


\section{Le Neo-royalisme: un combat d'avant-garde}

«Bien qu'on l'ait beaucoup dit, je ne suis pas né royaliste. Je ne suis même pas tout à fait un Blanc du Midi. La vérité, plus complexe, est aussi plus simple»23. C'est Maurras lui-même qui, en ouverture de son récit autobiographique de 1930 écrit pour dissiper les rumeurs autour d'une légende très répandue portant sur ses origines, ouvre à l'historien d'aujourd'hui les portes du malentendu développé à l'égard de la naissance de l'Action Française et de sa structure idéologique. L'absence pour le fondateur du néo-royalisme d'un héritage reçu «tout fait» par ses racines familiales, la nonappartenance à la Vendée provençale de Mistral (il était de Martigues, donc du Midi rouge, maritime ${ }^{24}$ ) et la présence d'un parcours individuel et pas du tout linéaire d'approchement aux idéaux royalistes, mettent déjà en évidence une élaboration originale, accomplie d'une façon très autonome par un jeune très doué, ayant en haine la République et la démocratie. Le nationalisme intégral maurrassien se fonde d'abord sur une hantise des origines: la radicalité du refus de l'héritage de 1789, Philippe Boutry nous l'a bien expliqué, est à la base du projet de régénération de l'idée de nation élaboré par Maurras dans le contexte passionnel de l'affaire Dreyfus. La Révolution était devenue pour lui, à la fois, quelque chose à nier et le symbole de tous les maux: mais le raisonnement qui l'avait emmené à son choix était bien loin de rassembler aux parcours traditionnels des «ultracistes» tout au long du siècle qu'allait terminer.

Le jeune Maurras développe, d'ailleurs, ses idées intellectuelles pendant la décennie qui va de la crise boulangiste à l'Affaire, et se nourrit de l'entrecroisement de thèmes dominants de la culture des nouvelles élites intellectuelles: «l'amertume de la défaite de 1870-1871, la hantise de la décadence, l'angoisse du déclassement, le désenchantement devant la République et face à la démocratie, l'obsession de la Revanche, la pénétration des idées antisémites et le mythe de l'armée» ${ }^{25}$. Cette dernière, incarnée pendant une étrange saison dans la figure éphémère du général Boulanger, et portée en triomphe par une coalition de défenseurs de l'ordre établi toujours plus hétéroclite, semblait avoir joué le rôle de fossoyeuse de la monarchie, ayant entraîné des conséquences

\footnotetext{
23 MAURRAS, Charles, Comme je suis devenu royaliste, paru dans La Revue de Paris en 1930, repris in ID., Au Signe de Flore, Paris, Les CEuvres Représentatives, 1931, p. 5.

${ }^{24}$ GOYET, Bruno, Charles Maurras, Paris, Presses de Sciences Po, 2000, p. 67.

25 BOUTRY, Philippe, L'Action Française, la Révolution et la Restauration, in LEYMARIE, Michel, PREVOTAT, Jacques (sous la direction de), L'Action Française, culture, société, politique, Paris, Editions du Septentrion, 2008, p. 28.
} 
catastrophiques sur le plan politique pour le milieu légitimiste français ${ }^{26}$. L'Armée et la Nation (ainsi que la fièvre antisémite, véritable raison mobilisatrice à droite) devenaient de plus en plus les catalyseurs des sentiments répandus de tous ceux qui s'opposaient à la République. Un potentiel révolutionnaire était prêt à se déclencher. Ce qui manquait, aux ennemis de la «Gueuse» c'était une même bataille à combattre, un cadre idéologique commun - qui devait comprendre des éléments anciens (conservatisme) en les fragmentant «avec irrespect des doctrines cohérentes» ${ }^{27}$ pour en obtenir un paradigme alternatif par rapport à celui mis en place par les républicains. Il fallait créer une nouvelle doctrine. Cela fut l'intuition de Charles Maurras.

Le jeune Maurras, pas encore royaliste, «à la recherche de ses principes» dans le Paris des années 1880, découvrait l'antisémitisme et l'amour pour le classicisme, voies par lesquelles il arrivera à un choix politique très claire pendant une époque marquée par la confusion: son premier vote aux élections de 1889 allait à la coalition boulangiste en nom de l'ordre conservateur, de l'honneur de l'Armée et de la Patrie. C'est seulement en 1896, d'après lui, que l'«aveu» de l'idée royaliste survient: dans le récit légendaire maurrassien, il s'agit d'une conversion qui se déchaîne pendant un voyage en Grèce, d'où il eut enfin la chance de regarder la France et ses malheurs de loin. Un an et demi plus tard, en plein Affaire Dreyfus, sa conversion devint effective. Aux yeux de ceux qui l'entouraient, Maurras était devenu royaliste surtout à cause du «péril soudain où la République jeta la Nation en cédant tout aux Juifs» ${ }^{28}$. Pour l'écrivain provençal et ses camarades, qu'il arrivera à convertir à l'idée royaliste en écrivant Dictateur et $R^{2}{ }^{29}$, la «conversion» a été un parcours tout à fait moderne, bien loin d'être la conséquence d'une foi héritée. Ils ont été conduits la monarchie par le nationalisme : Maurras, et après ses compagnons de route, se sont «découverts» monarchistes (tandis que les royalistes d'autrefois l'étaient de naissance) et ils resteront au fond toujours plus nationalistes que monarchistes et plus monarchistes que royalistes.

L'élaboration d'une nouvelle doctrine royaliste comportait, de l'autre côté, la perte (et la mise à jour) d'une partie de l'héritage contre-révolutionnaire pour longtemps admiré et placé au centre d'une quête de légitimité politique ${ }^{30}$. Le but de Maurras était celui de recréer les fondements de la monarchie en les adaptant à une forme spécifique

${ }^{26}$ Cfr. LEVILLAIN, Philippe, Boulanger, fossoyeur de la monarchie, Paris, Flammarion, 1982. ${ }^{27}$ NOLTE, Ernst, Le fascisme dans son époque: l'Action Française, Paris, Julliard, 1970, p. 289. 28 MAURRAS, Charles, De la politique naturelle au nationalisme intégral, Paris, Librairie Philosophique J. Vrin, 1972, pp. 37-48. 29 MAURRAS, Charles, Dictateur et Roi (1899), in Euvres capitales, Paris, Flammarion, 1954, pp. 250-285.

30 KUNTER, Tony, op. cit., pp. 42-43 
et moderne de l'idéologie nationaliste: pour lui l'État, la Nation, n'étaient plus ce qu'ils étaient pour Bonald, de droit divin, ils étaient devenus de droit naturel. «De Dieu à la nature, l'homme avait changé de maître. Il était toujours sujet» ${ }^{1}$. Ce type de conception nationaliste n'avait dès son début aucune similitude avec le vieux patriotisme d' État qui appartenait aux véritables légitimistes, aux partisans du Roi. «Son loyalisme profond, paradoxalement, ne allait pas au 'Pays', mais seulement à sa version particulière de ce Pays, construction idéologique fondée sur critères d'appartenance exclusifs : les Français sauf...»32. Une idée moderne de la France et de ses frontières (extérieures et intérieures), que les autres membres du cabinet du Roi, liés aux vieux schémas royalistes, avaient du mal à concevoir.

Certes, en épousant la cause du rétablissement de la monarchie, le mouvement s'attira inévitablement par esprit d'appartenance le soutien de ceux qui étaient affectivement attachés au royalisme et nostalgiques d'une époque révolue: pourtant, ça n’aurait évidemment pas suffi à faire de l'Action Française le point d'ancrage de tout anti-républicanisme, centre d'irradiation d'idées qui seront assimilées pendant les décennies suivantes par «des hommes de tous les bords»33. Le nationalisme intégral, en dépit des réductions avancées par la thèse dominante, était en réalité un système de pensée tout à fait moderne, où Monarchie et Église intervenaient en tant que conclusions et non en tant que fondements. Le message politique de l'AF, avant d'être une doctrine cohérente s'appuyant sur un mélange entre des longues traditions héritées, était d'abord une construction en négatif: il s'appuyait sur une critique radicale de la République et sur la thèse selon laquelle le Pays légal (la Troisième République) était l'antithèse du Pays réel (la vraie nation, dont la construction idéologique avait comme point d'ancrage la monarchie). Dans ce cadre, le nationalisme et l'antisémitisme (au sein duquel le nationalisme avait germé) étaient les vraies «raisons mobilisatrices»34, destinées à rassembler tous ceux qui s'opposaient au système de la Déclaration des droits de l'homme: des raisons puissantes sur lesquelles, en dernier lieu, le néo-royalisme s'appuyait en «habit de lumière». Cet apparent anachronisme, qui devait faire le succès des idées de l'Action Française, était, avant toute chose, une «arme idéologique propre à vaincre l'idéal républicain», un symbole même et garantie de l'unité nationale et de la cohésion sociale, l'étatisme républicain étant, selon la doctrine de l'AF, une conséquence naturelle de la religion démocratique.

${ }^{31}$ COLETTE CAPITAIN, Peter, op.cit., pp. 10-11.

$3^{2}$ GELLNER, Ernest, Nations et nationalismes, Paris, Payot, 1989, p. 175.

33 WEBER, Eugène, op. cit., p. 486.

34 GOYET, Bruno, op. cit., pp. 264-265. 
Ils combattaient les haïes «abstractions républicaines » avec un autre, complexe, système d'abstractions.

C'est à l'idée en soi de la royauté et pas à la personne du Roi qu'allait leur fidélité: héritiers des expériences nationalistes de la fin du siècle, la monarchie à laquelle les hommes de l'Action Française songeaient c'était précisément une monarchie apte à fonder cette nouvelle communauté imaginée, de nature idéologique et exclusive, une communauté que l'esprit antidémocratique et antirépublicain était capable de bâtir.

Si dans ce récit historique développé a posteriori, les ancêtres des BourbonsOrléans figuraient de fondateurs de l' État français moderne, c'était parce qu'ils étaient évoqués en tant que «preuves finales» de la légitimité historique de la doctrine maurrassienne, la maison royale ayant été reçue «toute faite de l'histoire de la Patrie»35. Les liaisons, parfois troublantes, entretenues avec les Prétendants à coup de désaveux royaux et de distinguos ${ }^{36}$ étaient, en effet, de plus en plus un indice de l'écart entre la monarchie-idée et la monarchie-réalité dans le nationalisme intégral. En théorisant une forme de «césarisme libéré de son caractère momentané»37 opposé aux principes démocratiques, les hommes de l'Action Française n'étaient guère disposés à jouer le rôle des simples partisans du Roi. Ils se plaçaient au-dessus du Roi lui-même, ils revendiquaient - en tant que Parti - la propriété intellectuelle et politique d'une conception restaurée de la royauté. «Mieux qu'à restaurer la Monarchie, l'Action Française cherchait en fait à instaurer sa propre monarchie» 38 .

Or, leur idée de nation, redéfinie selon les conceptions nées au tournant du XX siècle et fusionnée dans l'idée symbolique de la monarchie, était tout à fait un produit de la modernité, en tant que réaction à l'impact de l'essor de l'État moderne tel que nous l'a décrit Eric Hobsbawm: les bouleversements sociaux issus de l'industrialisation et de l'urbanisation rapides, l'avènement de l'ère de la politique de masse 39 . Les réalités, notamment, de la politique de masse et la nécessité de persuasion et de mobilisation étaient ainsi reconnues par l'Action Française sous de formes qui, avant 1914, en dehors des cercles socialistes et syndicalistes, étaient inédites et avancées: le but était celui de créer une contre-culture, une «contre-Encyclopédie», peu importait d'où les aspirants compagnons de route étaient issus. Nombre de ceux sur lesquels le mouvement exerça une influence étaient plus inspirés, au final, par ce à quoi il s'opposait que par ce qu'il

35 MAURRAS, Charles, Vingt-cinq ans de monarchisme, in ID., Oeuvres Capitales, t. II, Paris, Nouvelle librairie Nationale, 1925, p. 511.

36 WEBER, Eugène, op. cit., p. 81.

37 GURIAN, Waldemar, Der integrale nationalismus im Frankreich: Charles Maurras und die Action Française, V Klostermann, Frankfurt am Main, 1931, p. 92.

${ }^{38}$ COLETTE CAPITAIN, Peter, op. cit., pp. 33-40.

39 Cfr. HOBSBAWM, Eric, Nations et nationalismes depuis 1870, Paris, Gallimard, 1992. 
proposait: pour cette raison, l'Action Française peut être bien définie comme populistemoderne, «au sens où elle cherchait activement à conquérir une audience de masse, où elle savait flairer les thèmes et les questions susceptibles d'enflammer l'opinion publique et les exploiter d'une manière implacable et opportuniste»40. En dépit de l'étiquette de mouvement «élitiste-réactionnaire»41 qu'on lui a souvent attribué, elle a été capable d'être, à la fois, populaire et bien-pensant, populiste et élitiste, arrivant jusqu'à préfigurer un terrain d'entente avec les socialistes révolutionnaires par le biais de l'aventure du Cercle Proudhon ${ }^{42}$. Si cette dernière expérience finalement échoua, pourtant elle reste symptomatique par rapport au dépassement définitif du sectarisme royaliste de la part de l'Action Française et l'adhésion aux principes de l'ère des masses. Si on détourne le regard aux parcours des fascismes pendant leur montée au pouvoir, avec leur quête de légitimité auprès des vieilles élites ainsi que d'appui populaire et d'alliances peu orthodoxes, et si on abandonne la recherche d'une prétendue et rigide «linéarité» idéologique, on trouve un riche terrain de comparaison qui sort l'Action Française de toute référence stérile à un monde disparu. L'œuvre de l'historien serait celle de analyser une ère dans toute sans complexité, sans céder à la tentation d'une logique classificatoire et exclusive.

\section{Conclusion}

L'histoire de l'Action Française ouvre aux regards contemporains un chapitre symptomatique ainsi que fondamental de l'histoire d'un Pays qui entre 1789 et 1919 avait toujours été en avance de quelques pas sur le reste de l'Europe43: cette fusion des monarchistes nationaux, et des nationalistes royalistes avec les nationalistes républicains n'était pas vouée, par sa propre nature, à une existence éphémère. Le succès et l'influence obtenus partout dans la société française pendant 40 ans d'histoire, en dépit d'un manque d'exercice directe du pouvoir (sauf que pour certains d'entre eux sous Vichy) peuvent s'expliquer en effet par le fait que les activités de l'Action Française avaient bientôt attiré les mêmes forces (politiques et sociales) qui avaient été attirées par les appels des boulangistes et des antidreyfusards, des forces qui étaient destinée à marquer le paysage politique des décennies suivantes en passant par

40 JENKINS, Brian, op. cit., pp. 132, 136.

${ }^{41}$ Ibidem, p. 135.

42 Voir notamment, à propos du Cercle Proudhon et de son aventure éphémère pourtant significative, STERNHELL, Zeev, SZNAJDER, Mario, ASHERI, Maia, Naissance de l’idéologie fasciste, Paris, Folio, 2010; STERNHELL, Zeev, Ni droite ni gauche: l'idéologie fasciste en France, Paris, Fayard, 1983.

43 NOLTE, Ernst, op.cit., p. 55. 
les événements marquants et les transformations de début XXème siècle, ainsi que à travers les «baptêmes du feu» de la première guerre mondiale et de la Révolution bolchevique. C'est pour cette raison que, malgré l'accent mis sur l'importance du retour de la Couronne, l'influence de l'AF devait croître non pas à cause mais plutôt en dépit de cela; l'AF donnait un "point de ralliement» 44 aux couches sociales qui étaient attirées par les mots d'ordre de la droite et de l'extrême droite. Un projet politique sans doute avant-gardiste, qui envisageait faire trésor des expériences politiques du passé mais au même temps en dépasser les limites : la faute des nationalistes qui s'étaient succédés avant l'Action Française avait été, selon Maurras, celle de «viser le nombre, plus que l'énergie qu'il naît de la qualité et, avant tout, de la qualité des programmes», étant donné que munie d'une puissante doctrine «une poignée d'hommes avait fait la Révolution dans une France royaliste e catholique»45.

«Ceux qui placent les intérêts des partis au-dessus de la religion et veulent mettre la seconde au service des premiers»46: la condamnation pontificale de 1926, visant l'Action Française, aurait pu être sans doute revendiquée, lettre par lettre, par les prétendants de la Maison de France. Le royalisme, au final - autant que le catholicisme intégral - était une option de plus en plus symbolique ${ }^{47}$ pour le mouvement-revue, visant à donner une cohérence apparente à un système de pensée alternatif à celui de la République. Il s'agissait d'un principe fixé: «politique d'abord», et pas le Roi. Cette nouvelle révolution réactionnaire, d'ailleurs, pouvait se faire sans l'apport personnel du souverain : dans le nouveau monde des batailles idéologiques, la «royauté» n'avait guère besoin de son incarnation physique pour être fusionné avec la «véritable nation» dans le récit légendaire des origines nécessaire à tout nationalisme moderne. L'expérience de suspension des hostilités à l'égard de la République pendant la première guerre mondiale, ainsi que l'éphémère expérience parlementaire de l'Action Française, démontrent, s'il en avait encore besoin, qu'on était bien loin d'être face à un phénomène politique simple et prévisible. Lorsque en 1937 Maurras remarquait que désormais à cette époque-là « on ne craint plus l'accumulation des pouvoirs dans une seule main, mais on la désire [...] on ne dit plus que c'est injuste qu'un seul commande

44 WEBER, Eugène, op. cit., p. 71.

45 MAURRAS, Charles, L'énergie des minorités, cit. in JOLY, Bertrand, Les ligues nationalistes et l'Action Française, in LEYMARIE, Michel, PREVOTAT, Jacques (sous la direction de), L'Action Française, culture, société, politique, Paris, Editions du Septentrion, 2008, p. 96.

${ }^{4}$ DE LA MONTAGNE, Robert Harvard, Histoire de l'Action Française, Paris, Amiot-Dumont, 1950, p. 119.

47 JENKINS, Brian, op. cit., pp. 130-135. 
à tous les autres»48 il reconnaissait sans doute que ce résultat, la naissance d'un fort désir d'autorité diffusé dans la société française et d'un esprit «non-conformiste» à l'égard de l'héritage de 1789, avait été en part le produit de l'œuvre méticuleuse de vidage des valeurs républicains et démocratiques accomplie par son organisation pendant des décennies. Le «coup de force» culturel avait finalement atteint son but. Le Roi tant attendu aurait eu, trois années plus tard, le visage rassurant d'un vieux maréchal conservateur.

48 MAURRAS, Charles, Vingt-cinq ans de monarchisme, in Euvres Capitales, t. II, Paris, Flammarion, 1954, pp. 490-491. 


\section{* L'auteur}

Andrea Cavaletto, diplômé de l'Université de Turin, est doctorant en histoire contemporaine auprès de l'Université Lumière Lyon 2, rattaché au LAboratoire de Recherches Historiques Rhône-Alpes (LARHRA). Sa thèse, dirigée par Laurent Douzou, porte sur l'évolution de la droite française entre les années 1930 et le régime de Vichy.

URL: < http://www.studistorici.com/progett/autori/\#Cavaletto >

\section{Per citare questo articolo:}

CAVALETTO, Andrea, «La monarchie imaginée: sur le royalisme dans l'idéologie de l'Action Française», Diacronie. Studi di Storia Contemporanea : Le monarchie nell'età dei nazionalismi, 29/12/2013,

URL: < http://www.studistorici.com/2013/12/29/cavaletto_numero_16/ >

Diacronie Studi di Storia Contemporanea $\vartheta$ www.diacronie.it

Risorsa digitale indipendente a carattere storiografico. Uscita trimestrale. redazione.diacronie@hotmail.it

Comitato di redazione: Marco Abram - Jacopo Bassi - Luca Bufarale - Alessandro Cattunar - Elisa Grandi - Deborah Paci - Fausto Pietrancosta - Matteo Tomasoni - Luca Zuccolo 